\title{
Copy Number Assessment of SMN1 Based on Real- Time PCR With High-Resolution Melting: Fast and Highly Reliable Testing
}

\section{Ying Xu}

Xijing Hospital, Fourth Military Medical University

\section{Tingting Song}

Xijing Hospital, Fourth Military Medical University

\section{Xiaozhou Wang}

Xi'an Tianlong Science and Technology

Jiao Zheng

Xijing Hospital, Fourth Military Medical University

Yu Li

Xijing Hospital, Fourth Military Medical University

\section{Fenfen Guo}

Xijing Hospital, Fourth Military Medical University

\section{Yuanfeng Li}

Xijing Hospital, Fourth Military Medical University

\section{Zijian Guo}

Peking Union Medical College Hospital, Chinese Academy of Medical Science

\section{Yaling Dou}

Peking Union Medical College Hospital, Chinese Academy of Medical Science

\section{Yu Wang}

Xi'an Tianlong Science and Technology

\section{Ye Zhao}

Xi'an Tianlong Science and Technology

Hong Yang ( $\nabla$ yanghongfck@163.com )

Xijing Hospital, Fourth Military Medical University

\section{Research Article}

Keywords: Spinal muscular atrophy, SMN1, High-resolution melting, carrier screening

Posted Date: September 7th, 2021

DOI: https://doi.org/10.21203/rs.3.rs-863046/v1 
License: (c) (i) This work is licensed under a Creative Commons Attribution 4.0 International License. Read Full License

Version of Record: A version of this preprint was published at Brain and Development on April 1st, 2022. See the published version at https://doi.org/10.1016/j.braindev.2022.03.011. 


\section{Copy Number Assessment of SMN1 based on Real-Time PCR with High-Resolution Melting: Fast and Highly Reliable Testing}

Ying $\mathrm{Xu}^{1}$, Tingting Song ${ }^{1}$, Xiaozhou Wang ${ }^{2}$, Jiao Zheng ${ }^{1}$, Yu $\mathrm{Li}^{1}$, Fenfen Guo ${ }^{1}$, Yuanfeng $\mathrm{Li}^{1}$, Zijian Guo ${ }^{3}$, Yaling $\mathrm{Dou}^{3}$, Yu Wang ${ }^{2}$, Ye Zhao ${ }^{2} *$ and Hong Yang ${ }^{1}$.

${ }^{1}$ Department of Obstetrics and Gynecology, Xijing Hospital, Fourth Military Medical University, Shannxi 710032, China

${ }^{2}$ Xi'an Tianlong Science and Technology CO.,Ltd

${ }^{3}$ Peking Union Medical College Hospital, Chinese Academy of Medical Sciences

*Correspondence: Hong Yang \& Ye Zhao

yanghongfck@163.com \& zhaoye708910614@126.com

Department of Obstetrics and Gynecology, Xijing Hospital, Fourth Military Medical University, No.127 Changle Road (West), XinCheng District, Xi’an, Shannxi, China, 710032. 


\begin{abstract}
Background: Spinal muscular atrophy (SMA) is a common neuromuscular disorder, caused by absence of both copies of the survival motor neuron 1 (SMN1) gene. Population-wide SMA screening to quantify copy number of SMN1 is recommended by multiple regions. SMN1 diagnostic assay with simplified procedure, high sensitivity and throughput is still needed.

Methods: Real-Time PCR with High-Resolution Melting for the quantification of the SMN1 gene exon 7 copies and SMN1 gene exon 8 copies was established and confirmed by multiplex ligation-dependent probe amplification (MLPA). The diagnosis of 2563 individuals including SMA patients, suspected cases and the general population were analyzed by the real-time PCR. The results were compared with the gold standard test MPLA.

Results: In this study, the homozygous deletions, heterozygous deletions were identified by Real-Time PCR with High-Resolution Melting method with an incidence of $10.18 \%$ and $2.42 \%$, respectively. In addition, the $\mathrm{R}$ value distribution $(\mathrm{P}>0.05)$ among the 8 replicates and the coefficient of variation $(\mathrm{CV}<0.003)$ suggested that the qPCR screening test had high reproducibility. High concordance was obtained between Real-Time PCR with High-Resolution Melting and MPLA.
\end{abstract}

Conclusions: The qPCR based on High-Resolution Melting provides a sensitive and high-throughput approach to large-scale SMA carrier screening with low cost and labor.

Keywords: Spinal muscular atrophy; SMN1; High-resolution melting; carrier screening

\title{
Introduction
}

Spinal muscular atrophy (SMA) is one of the most common recessive neuromuscular disorders, which is always accompanied with progressive muscle atrophy leading to respiratory failure[1]. Based on the age of onset and clinical severity, SMA is classified into four clinical types: onset at 0-6 months of age, unable to sit unaided (type I), onset at $<18$ months of age able to sit but unable to walk independently (type II), onset at>18 months of age with weaker legs than arms (type III), and adult onset SMA (type IV)[2].

SMA is an incurable autosomal recessive motor neurone disease, which caused by mutation of the motor neuron survival gene SMN located at chromosome 5q13[3]. As the SMA-related genes, the two genes are identical except for five nucleotides (SMN2 differs from SMN1 by only five nucleotides), but they play different roles in SMA disease respectively. SMA occur secondary to 
alterations in the SMN1gene, which is the main driver of SMN protein production. The severity of the disease is determined by the number of copies of the SMN2 gene, which is a homolog to SMN1 but not as efficient in protein production[4, 5]. Studies have shown that more than $90 \%$ of SMA patients have homozygous deletion of SMN1 gene, and the rest are heterozygous deletion, point mutation or transformation of SMN1 gene into SMN2 gene[6].

SMA occurs in approximately 1 in 10,000 or 6000 live births with a carrier frequency of 1 in 50 or 40[7]. Considering the potentially severe phenotype and the high prevalence of SMA, population-wide SMA screening is recommended by the American College of Medical Genetics and Genomics[8, 9]. The copy number of SMN1 for SMA diagnosis and carrier testing (in either the pre-conception or prenatal period) is proved to be beneficial, so that an available and clinically valid screening method is needed for determination of the SMN1 copy number[10].

There are methods for quantitative analysis of the SMN copies. Multiplex ligation-dependent probe amplification (MLPA), which is generally acknowledged as a gold standard for determining SMA carriers with high degree of precision for the quantification of SMN1 copy number, and the probe combination can detect both exons 7 and 8 of the SMN1 and SMN2[11, 12]. Due to multiple steps, MLPA always requires a long turnaround time and relatively high costs. The Denaturing high performance liquid chromatography (DHPLC) could be used to detect the copies of the SMN gene by comparing the peak of amplified product with the reference, but is labor highly demanding detection environment $[13,14]$. Next-generation sequencing (NGS) requires relative high sequencing depth to obtain accurate carrier gene, which need deep bioinformation analysis and increases the costs[15]. High resolution melting analysis (HRMA) was applied to SMA detection which has been demonstrated in recent studies. HRMA is a powerful post-PCR technique which reduces detection costs, simplifies operational steps by melting curve with saturation LC Green dyes. So HRMA has a great potential in SMA large-scale population screening[16, 17]. Digital droplet PCR (ddPCR) has great specificity for nucleotide target direction because amplification and signals collection were performed within each droplet, but digital PCR and liquid bead array is relatively costly and rarely used in underdeveloped regions[18]. Real-time Quantitative PCR (qPCR) is easy to perform with low cost and labor, which can be used for large-scale mutation screening in clinical laboratories. qPCR with melting curve analysis require no post-PCR manipulations, thereby minimizing the risk 
of cross contamination[19]. Thus qPCR is low cost and easy to design due to no need for special fluorescent probes, which would be an ideal platform for carrier screening of SMA.

In the present study, the qPCR method based on High-Resolution Melting was carred out to determine the copy number of SMN1 from 2563 clinical samples. Furthermore, the reliability and stability of the method were evaluated by comparing the quantitative results of SMN1 genes with MLPA assay. Thus, our data uncovered an important role of qPCR in rapid SMA screening.

\section{Result}

\section{qPCR Based On High-Resolution Melting for SMN1 CNV Assessment}

We developed SMN1 copy number screening a qPCR-basee metlting cure exon 7 and exon 8 of SMN1 can be analyzed simultaneously by qPCR method, and exon 7 and CFTR gene of SMN1 can be amplified simultaneously. The melting curve of exon 7 of SMN1 and CFTR gene is shown in Figure. 1A. Melting peaks of CFTR and SMN1 exon 7 are $72.5^{\circ} \mathrm{C}$ and $77^{\circ} \mathrm{C}$, respectively. Similarly, the melting peaks of exon 8 of SMN1 and TPGS2 were $78^{\circ} \mathrm{C}$ and $83^{\circ} \mathrm{C}$, respectively (Figure.1B). The data indicated that the SMN1 melting peak can be clearly distinguished from that of the internal reference gene. We can assess the copy number of the SMN1 based on the R value of the peaks combined with the control results. The results showed that only when the corresponding sample copy number existed in the reaction, the SMN1 copy number had the specific signal, and the analysis specificity was $100 \%$.

\section{Reproducibility of the qPCR method}

One or two copies of exon 7 and exon 8 of SMN1 gene were detected by eight repeated qPCR methods at different time points. The $\mathrm{R}$ value was calculated to evaluate the reproducibility of the qPCR test. As shown in Table 1 , the $\mathrm{R}$ value distribution $(\mathrm{P}>0.05)$ among the 8 replicates and the coefficient of variation $(\mathrm{CV})<0.003$ suggested that the qPCR screening test had high reproducibility.

\section{Clinical performance of $q P C R$ compared with MLPA}

In this study, a total of 2563 samples were successfully genotyped by qPCR. 10.26\% (263/2563) of the participants had 0 copy exon 7 of SMN1 gene. $2.15 \%(55 / 2563)$ of the participants had 1 copy and $87.59 \%(2245 / 2563)$ of the participants had 2 or more copies exon 7 of SMN1 gene. The results were consistent with those of MPLA (Table S2). In addition, for exon 8 of SMN1 gene, the results of qPCR and MLPA were consistent, 9.29\%(238/2563) of participants had a copy number of 0,3.28\% 
(84/2563) of participants had a copy number of $1,87.43 \%(2241 / 2563)$ of participants had a copy number of 2 or above(Table S3). The sensitivity and specificity of the SMN1 qPCR method to demonstrate that the two platforms could accurately determine the copy number of exons 7 and 8 were $100 \%$ for SMN1 exons 7 and 8. The Cohn Kappa coefficient between the qPCR test and MLPA was 1.

\section{SMA screening results of clinical samples}

In the melting profiles of 2563 clinical sample, the classic homozygous deletion of SMN1 exon 7 accounted for 261 (10.18\%), heterozygous mutations for $62(2.42 \%)$, and $\geq 2$ copies of SMN1 exon 7 accounted for $2240(87.40 \%)$. The results showed that the frequency of copy number variation in exon 7 of SMN1 gene was similar to that in exon 8, and most of the exons 7 and 8 of SMN1 gene were homozygous or heterozygous deletion (Table 2). We further analyzed the detection frequency of different groups. As shown in Table 3, the incidence of homozygous and heterozygous deletion of SMN1 was slightly higher in patients with suspected SMA (about 4.33\%), and mutations in exon 7 of SMN1 accounted for $2.1 \%$ in the normally screened population. Exon 8 copy number variation showed a similar trend.

In addition, we summarized the rate of heterozygous deletion of SMN1 exon 7 in the screening cases from four regional hospitals. The SMA carrier rates of Xijing Hospital, The Second Affiliated Hospital of Zhejiang University Medical College, Peking Union Medical Hospital and The First Affiliated Hospital of Fujian Medical University were $1.67 \%, 2.33 \%, 1.33 \%$ and $2.36 \%$, respectively (Figure 2).

\section{Discussion}

As one of the leading genetic cause of infant death, SMA is a common neuromuscular disorder caused by mutations in SMN1, which has been considered an incurable disease[20, 21]. Given the severity of the disease and the relatively high frequency of SMA in the general population, an accurate and early screening technology that can identify SMN1 deletion and carriers is needed. In addition, for screening applications involving a large number of samples, such as active SMA screenings for diagnostic assay can be a challenge as both the throughput and cost of the assay have to be considered[9]. In this study, we describe a high-throughput, accurate and quantitative molecular approach based on qPCR. The qPCR method not only can detect homozygous deletion of SMN1, but 
also can used semi-quantitative analysis to identify the heterozygous mutation of the pathogenic gene of the carrier so that achieving SMA carrier screening.

Although absolute quantification method with probe is preferred due to more specific than comparative quantification, but requires quantitative external reference samples with complex probe design and increased costs[19, 22]. The qPCR method is based on high-resolution melting for comparative quantification, and only a two-copy SMN1 reference is needed for calculating $\mathrm{R}$ value to conclude the copy number of SMN1. We can see the copy number of the SMN1 directly from the melting curve (as shown in Fig.1). For exon 7 and exon 8 of SMN1 mutation assay, our qPCR method based on High-Resolution Melting showed the preferable reproducibility. To verify the reliability of the qPCR method based on High-Resolution Melting, we applied the method to the active SMN1 mutation screening of 2563 clinical samples from 4 hospitals and compared the results with standard MLPA. Results show both MLPA and our qPCR methods agree in diagnosis for SMN1 homozygous deletion variants and heterozygous deletion carriers. A total of 263 individuals were found to be homozygous deletion of SMN1, 55 carriers with SMN1 heterozygous mutation and 2245 individuals with $\geq 2$ copies SMN1, and consistent results of exon 8 were obtained from MLPA and qPCR. All of results showed the $100 \%$ sensitivity and $100 \%$ specificity of the qPCR based on High-Resolution Melting compared with MLPA. The kappa coefficient (100\%) showed the results of these two methods have no statistical differences, which proved the qPCR method were able to accurately determine the copy number of exon 7 and exon 8 of the SMN1 gene.

Current reports show that about $95 \%$ of patients with SMA are due to deletion of exon 7 or deletion of exons 7 and 8 simultaneously in the SMN1 gene, while the remaining 5\% are due to the simultaneous presence of deletion mutations and point mutations in the SMN1 gene[23]. In this study, total 363 SMA patients were analyzed for detecting deletion variants in patients with SMA. We found that the proportion of both exon 7 and exon 8 of SMN1 simultaneously homozygous deletion was high $(89.35 \%)$, while only exon 7 of the SMN1 gene was homozygous deletion in $9.50 \%$ of SMA patients. The results that exon 7 had a higher deletion rate than exon 8 in SMA patients were consistent with other reports, which showed that the high correlation of exon 7 of the SMN1 gene with the SMA[5, 23]. In addition, for 300 suspected SMA patients, there were 3 individuals with homozygous deletion of exon 7 of SMN1, and the slightly higher frequency heterozygous deletion of SMN1 in the suspected SMA patients (about 3.33\%), further illustrates that 
SMN1 exon 7 deletions account for most DNA alterations leading to SMA[24, 25]. Total 2000 normal screening participants from 4 hospitals were assayed by qPCR based on High-Resolution Melting, and among them there were 42 carriers with heterozygous deletion of exon 7 of SMN1 (2.1\%), which were similar to most reports. All of 4 hospitals had SMA carriers in our study, the proportion is from $1.33 \%-2.5 \%$ (Fig. 2). Due to participants from various regions, the different proportion of carriers in 4 hospitals, which is often reported that different prevalence of carrier genotypes appears in different ethnic populations[26, 27], showed the mutation detection of SMN1 plays a crucial role in SMA carrier screening.

Considering the practicability of mutation detection of SMN1 exon 7 and exon 8 for SMA, our method aims to provides an alternative method based on qPCR platform to advance the implementation of a carrier screening program for SMA.

In conclusion, we have developed a high-throughput and simple screening technology based on High-Resolution Melting on qPCR platform. Our qPCR method was 100\% agreement with the MLPA, which showed highly sensitive (100\%) and specific (100\%). The assay in general can be used for large-scale SMA carrier screening as a highly-sensitive and high-throughput approach with low cost and labor.

\section{Methods}

\section{Clinical samples}

The blood samples were collected from 4 hospitals: Xijing Hospital, The Second Affiliated Hospital of Zhejiang University Medical College, Peking Union Medical Hospital and The First Affiliated Hospital of Fujian Medical University. A total of 2563 individuals (include SMA patients, clinically suspected cases and normal screening person) were enrolled in this study. Clinically suspected cases involve high creatine kinase, Facial shoulder and arm muscular dystrophy, Weakness of the lower limbs, Lower extremity muscle atrophy, congenital myopathy, congenital myasthenia syndrome and other clinical symptoms similar to SMA. Normal SMA screening person include pregnant women (at various months of pregnancy 5 weeks, 10 to 32 weeks, 34 to 37 weeks, and 39 weeks) and normal healthy individuals. The general characteristics are provided in Table S1. Ethical approval for the study were approved by the institutional review boards of the Xijing Hospital (Approval no. KY20203041-1), The Second Affiliated Hospital of Zhejiang University Medical 
College (Approval no. 2019-003), Peking Union Hospital (Approval no. KS2019412) and The First Affiliated Hospital of Fujian Medical University (Approval no. LS2019-003-01). All the subjects signed the informed consent. All procedures performed in studies involving human participants were in accordance with the ethical standards of the institutional and/or national research committee and with the 1964 Helsinki declaration and its later amendments or comparable ethical standards.

\section{DNA extraction}

The genomic DNA was extracted from whole blood samples with the Ex-DNA Blood DNA Kits (TIANLONG, CHINA) according to the manufacturer's instructions. The extracted DNA was dissolved in $50 \mu \mathrm{L}$ ddH2O and quantified with NanoDrop (Thermo Fisher, USA). DNA concentration that less than $10 \mathrm{ng} / \mu \mathrm{l}$ needs to be re-extracted, whereas higher than $120 \mathrm{ng} / \mu \mathrm{l} \mathrm{DNA}$ needs to dilute to an appropriate concentration $(10 \mathrm{ng} / \mu \mathrm{l}-120 \mathrm{ng} / \mu \mathrm{l})$ before testing.

\section{qPCR Based on High-Resolution Melting}

Two multiplex qPCR reaction were performed to determinate the copy number of exon 7 and exon 8 of SMN1 simultaneously. As an internal reference, CFTR and TPGS2 as an internal reference were separately used to determine the effectiveness of the amplification. A total of $20 \mu \mathrm{L} q \mathrm{PCR}$ reaction mixture containing $2 \mu \mathrm{L}$ genomic DNA, 1X SMN1-7 or SMN1-8 Buffer (TIANLONG, CHINA) and 500nm/L of each primer, 1U KlenTaq1TM (Ab Peptides) . Also included were $1 \mathrm{X}$ LCGreen ${ }^{\circledR}$ Plus dye (BioFire Defense). The multiplex PCR was performed on a Light Cycler® 480 Real-Time PCR System (Roche) with the following thermal profile: $95^{\circ} \mathrm{C}$ for $5 \mathrm{~min}, 10$ cycles of $95{ }^{\circ} \mathrm{C}$ for $5 \mathrm{~s}$, touchdown $64^{\circ} \mathrm{C}$ to $60{ }^{\circ} \mathrm{C}$ for 30 s, then 25 cycles of $95{ }^{\circ} \mathrm{C}$ for $10 \mathrm{~s}, 60{ }^{\circ} \mathrm{C}$ for $15 \mathrm{~s}$. After amplification, the the samples were heated to $95{ }^{\circ} \mathrm{C}$ for $60 \mathrm{~s}$, cooled to $40{ }^{\circ} \mathrm{C}$ for $60 \mathrm{~s}$, and then melted from $65{ }^{\circ} \mathrm{C}$ to $95{ }^{\circ} \mathrm{C}$ using 25 acquisitions $/{ }^{\circ} \mathrm{C}$ to acquire high-resolution melting data. DNA samples which had been confirmed to contain zero, one copies of SMN1 and two copies of SMN1 were used as controls, of which two copies of SMN1 were also used as standard.

\section{MLPA assay}

MPLA provided further validation for the cases of SMA. The MLPA products were detected using ABI PRISM 3730XL sequencer (Applied Biosystems, USA) and analyzed using 
Coffalyser.Net software (MRC Holland). The detailed methods and primer needs were obtained as described in the previous literature.

\section{Statistical analysis}

Variation HRM Analysis Software (V1.0) was used to analyze the copy number of SMN1 exon 7 and exon 8. We need two parameters: 1) P value represents the melting peak height of the SMN1 gene. If $\mathrm{P}$ is equal to 0 , the copy number of SMN1 gene exon 7 (exon 8 ) of the sample is 0 , which is a homozygous deletion. 2) $\mathrm{R}$ value represents the ratio of the melting peak of the SMN1 gene to the melting peak of the control with 2 copies of SMN1. Different ranges of R values represents SMN1 copy number: (1)one copy with heterogeneous deletion $(\mathrm{R} \leq 0.70)$; (2) Two copies or more $(\mathrm{R} \geq 0.74)$; (3)Repeat the test again $(0.70<\mathrm{R}<0.74)$. Statistical analysis was performed using GraphPad Prism 8 (GraphPad software). The comparison between two groups was performed using unpaired Student's t-test by SPSS version 16 software (IBM Corp., USA). A value of $\mathrm{P}<0.05$ was considered statistically significant. The statistical tests used were two-sided.

\section{Acknowledgements}

We are grateful for the valuable data with the Second Affiliated Hospital of Zhejiang University Medical College, The first affiliated hospital of Fujian Medical University and Peking Union Medical college hospital.

\section{Funding}

This work was funded by the Sub-Project National Key Scientific Instruments and Equipment Development Project of China (2018YFF01012100) and Xi'an Science and Technology Project (20YXYJ0009-7).

\section{Author Contributions}

Y.Z. and H.Y. conceived and designed the study. Y.X. drafted the manuscript. T.S. and F.G contributed to collect the samples. Y.X., T.S., X.W., J.Z., Y.L., Z.G and Y.D. performed the experiments. YF.L., F.G, and Y.W. contributed to the data analysis. Y.Z. revised the grammar of the manuscript. H. Y. reviewed and edited the manuscript.

\section{Competing interests}

The author(s) declare no competing interests.

\section{Reference}

[1] Ross LF, Kwon JM, Spinal Muscular Atrophy: Past, Present, and Future. Neoreviews. 20, 
e437-e451 (2019).

[2] Wijaya YOS, Nishio H, Niba ETE, Shiroshita T, Kato M, Bouike Y et al., Dried Blood Spot Screening System for Spinal Muscular Atrophy with Allele-Specific Polymerase Chain Reaction and Melting Peak Analysis. Genet Test Mol Biomarkers. 25, 293-301 (2021).

[3] Arnold ES, Fischbeck KH, Spinal muscular atrophy. Handb Clin Neurol. 148, 591-601 (2018).

[4] Bharucha-Goebel D, Kaufmann P, Treatment Advances in Spinal Muscular Atrophy. Curr Neurol Neurosci Rep. 17, 91 (2017).

[5] Calucho M, Bernal S, Alias L, March F, Vencesla A, Rodriguez-Alvarez FJ et al., Correlation between SMA type and SMN2 copy number revisited: An analysis of 625 unrelated Spanish patients and a compilation of 2834 reported cases. Neuromuscul Disord. 28, 208-215 (2018).

[6] Muralidharan K, Wilson RB, Ogino S, Nagan N, Curtis C, Schrijver I, Population carrier screening for spinal muscular atrophy a position statement of the association for molecular pathology. J Mol Diagn. 13, 3-6 (2011).

[7] Sugarman EA, Nagan N, Zhu H, Akmaev VR, Zhou Z, Rohlfs EM et al., Pan-ethnic carrier screening and prenatal diagnosis for spinal muscular atrophy: clinical laboratory analysis of >72,400 specimens. Eur J Hum Genet. 20, 27-32 (2012).

[8] Rouzier C, Chaussenot A, Paquis-Flucklinger V, Molecular diagnosis and genetic counseling for spinal muscular atrophy (SMA). Arch Pediatr. 27, 7S9-7S14 (2020).

[9] Prior TW, Professional P, Guidelines C, Carrier screening for spinal muscular atrophy. Genet Med. 10, 840-842 (2008).

[10] Deignan JL, Astbury C, Behlmann A, Guha S, Monaghan KG, Reddi HV et al., Addendum: Technical standards and guidelines for spinal muscular atrophy testing. Genet Med. 10.1038/s41436-020-00961-6 (2020).

[11] Alias L, Bernal S, Barcelo MJ, Also-Rallo E, Martinez-Hernandez R, Rodriguez-Alvarez FJ et al., Accuracy of marker analysis, quantitative real-time polymerase chain reaction, and multiple ligation-dependent probe amplification to determine SMN2 copy number in patients with spinal muscular atrophy. Genet Test Mol Biomarkers. 15, 587-594 (2011).

[12] Hwang H, Lee JH, Choi YC, Clinical Characteristics of Spinal Muscular Atrophy in Korea Confirmed by Genetic Analysis. Yonsei Med J. 58, 1051-1054 (2017). 
[13] Tan Y, Wang H, Zhao T, Cheng M, Zhang C, [Supplementary value of denaturing high performance liquid chromatography for routine prenatal diagnosis of spinal muscular atrophy by multiple ligation-dependent probe amplification]. Zhonghua Yi Xue Yi Chuan Xue Za Zhi. 36, 1175-1178 (2019).

[14] Shaw SW, Cheng PJ, Chang SD, Lin YT, Hung CC, Chen CP et al., Rapid prenatal diagnosis of spinal muscular atrophy by denaturing high-performance liquid chromatography system. Acta Obstet Gynecol Scand. 87, 960-968 (2008).

[15] Lopez-Lopez D, Loucera C, Carmona R, Aquino V, Salgado J, Pasalodos S et al., SMN1 copy-number and sequence variant analysis from next-generation sequencing data. Hum Mutat. 41, 2073-2077 (2020).

[16] Zhou L, Palais RA, Paxton CN, Geiersbach KB, Wittwer CT, Copy number assessment by competitive PCR with limiting deoxynucleotide triphosphates and high-resolution melting. Clin Chem. 61, 724-733 (2015).

[17] Wang KC, Chang CC, Chang YF, Wang SH, Chiang CK, Tsai CP, Evaluation and characterization of a high-resolution melting analysis kit for rapid carrier-screening test of spinal muscular atrophy. J Neurogenet. 29, 113-116 (2015).

[18] Stabley DL, Holbrook J, Scavina M, Crawford TO, Swoboda KJ, Robbins KM et al., Detection of SMN1 to SMN2 gene conversion events and partial SMN1 gene deletions using array digital PCR. Neurogenetics. 22, 53-64 (2021).

[19] Maranda B, Fan L, Soucy JF, Simard L, Mitchell GA, Spinal muscular atrophy: clinical validation of a single-tube multiplex real time PCR assay for determination of SMN1 and SMN2 copy numbers. Clin Biochem. 45, 88-91 (2012).

[20] Minino AM, Xu J, Kochanek KD, Deaths: preliminary data for 2008. Natl Vital Stat Rep. 59, $1-52(2010)$.

[21] Arnold WD, Kassar D, Kissel JT, Spinal muscular atrophy: diagnosis and management in a new therapeutic era. Muscle Nerve. 51, 157-167 (2015).

[22] Xia Z, Zhou Y, Fu D, Wang Z, Ge Y, Ren J et al., Carrier screening for spinal muscular atrophy with a simple test based on melting analysis. J Hum Genet. 64, 387-396 (2019).

[23] Wirth B, An update of the mutation spectrum of the survival motor neuron gene (SMN1) in autosomal recessive spinal muscular atrophy (SMA). Hum Mutat. 15, 228-237 (2000). 
[24] Laird AS, Mackovski N, Rinkwitz S, Becker TS, Giacomotto J, Tissue-specific models of spinal muscular atrophy confirm a critical role of SMN in motor neurons from embryonic to adult stages. Hum Mol Genet. 25, 1728-1738 (2016).

[25] Zapletalova E, Hedvicakova P, Kozak L, Vondracek P, Gaillyova R, Marikova T et al., Analysis of point mutations in the SMN1 gene in SMA patients bearing a single SMN1 copy. Neuromuscul Disord. 17, 476-481 (2007).

[26] Zhang J, Wang Y, Ma D, Sun Y, Li Y, Yang P et al., Carrier Screening and Prenatal Diagnosis for Spinal Muscular Atrophy in 13,069 Chinese Pregnant Women. J Mol Diagn. 22, 817-822 (2020).

[27] Su YN, Hung CC, Lin SY, Chen FY, Chern JP, Tsai C et al., Carrier screening for spinal muscular atrophy (SMA) in 107,611 pregnant women during the period 2005-2009: a prospective population-based cohort study. PLoS One. 6, e17067 (2011).

\section{Figure legends}

\section{Fig. 1 melting profiles derived from qPCR assay}

Melting curve and melting peak analysis of SMN1, (A) Melting analysis of multiplex PCR of SMN1 exon 7/ CFTR, (B)Melting analysis of multiplex PCR of SMN1 exon 8/TPGS2. There were no unexpected amplification products with different melting curves, the height of melting curve points to different copy number of SMN1.

Fig. 2 The prevalence of carriers in normal screening group from 4 hospitals

The rate of heterozygous deletion of SMN1 exon 7 in the screening cases from different regional hospitals were plotted and compared. 


\section{Figures}
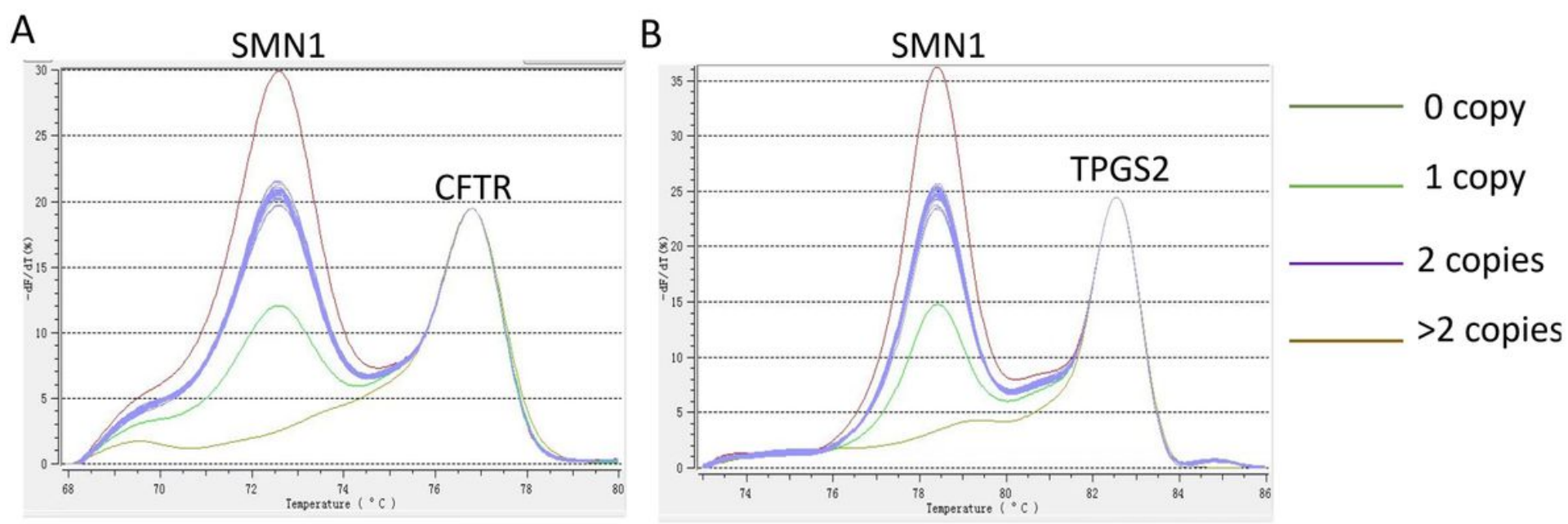

Figure 1

melting profiles derived from qPCR assay Melting curve and melting peak analysis of SMN1, (A) Melting analysis of multiplex PCR of SMN1 exon 7/ CFTR, (B)Melting analysis of multiplex PCR of SMN1 exon 8/TPGS2. There were no unexpected amplification products with different melting curves, the height of melting curve points to different copy number of SMN1.

The Second Affiliated Hospital of Zhejiang University Medical College-

1.67

The first Affiliated Hospital of Fujian Medical University-

Peking Union Medical College HospitalXijing Hospital
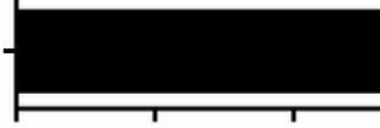

1.33

0.
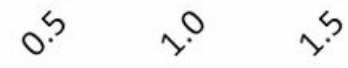

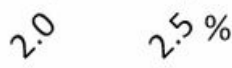

Carrier Proportion

\section{Figure 2}

The prevalence of carriers in normal screening group from 4 hospitals The rate of heterozygous deletion of SMN1 exon 7 in the screening cases from different regional hospitals were plotted and compared.

\section{Supplementary Files}

This is a list of supplementary files associated with this preprint. Click to download. 
- Tables1.pdf

- Tables.pdf 